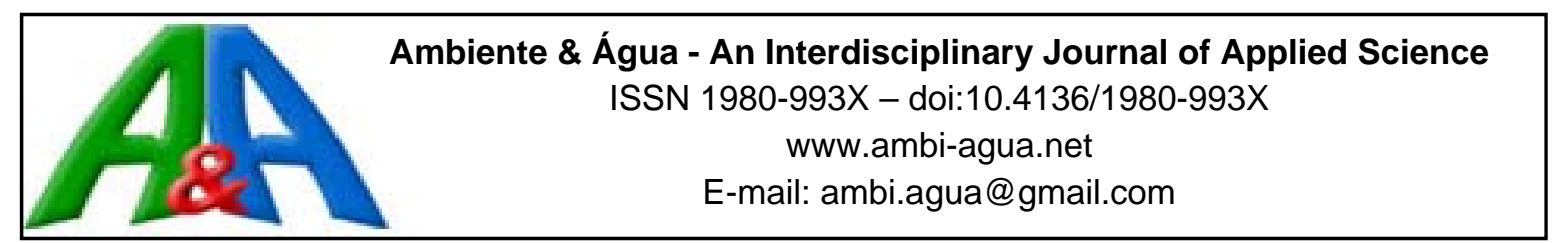

\title{
Teores de Arsênio em solos de três regiões do estado de Santa Catarina
}

\author{
doi:10.4136/ambi-agua.1746
}

Received: 03 Sep. 2015; Accepted: 17 Dec. 2015

\author{
Leticia Cristina de Souza $^{1 *}$; Mari Lucia Campos ${ }^{1}$; Gabriela Reichert ${ }^{2}$; \\ Carolina Natel de Moura ${ }^{3}$ \\ ${ }^{1}$ Universidade do Estado de Santa Catarina (UDESC), Lages, SC, Brasil \\ Departamento de Solos e Recursos Naturais \\ ${ }^{2}$ Universidade Federal do Paraná (UFPR), Curitiba, PR, Brasil \\ Departamento de Hidráulica e Saneamento \\ ${ }^{3}$ Universidade do Estado de Santa Catarina (UDESC), Lages, SC, Brasil \\ Departamento de Engenharia Ambiental \\ *Autor correspondente: e-mail: leti_quimica@yahoo.com.br, \\ mari.lucia03@gmail.com, gabrielareichertamb@gmail.com, \\ carolina.natel@hotmail.com
}

\section{RESUMO}

A determinação do teor natural de elementos-traço é essencial para monitorar a entrada de tais elementos no sistema solo e contribuir na remediação de áreas contaminadas. $\mathrm{O}$ objetivo desse trabalho foi determinar o teor natural de Arsênio em solos provenientes de três regiões do estado de Santa Catarina: Planalto Sul, Metropolitana e Litoral Sul. O teor de Arsênio foi obtido após digestão em forno de micro-ondas, seguindo o protocolo da USEPA 3051 A e quantificação em espectrometria de absorção atômica com atomização eletrotérmica. Os resultados foram analisados pelo teste Scott-Knott a 5\% de significância. Os atributos do solo que melhor se correlacionaram com os teores de Arsênio foram argila, carbono orgânico, capacidade de troca de cátions e óxidos de alumínio e de ferro. Os teores de Arsênio estão relacionados ao material de origem e ao relevo dos solos das regiões.

Palavras-chave: áreas contaminadas, elementos-traço, remediação.

\section{Arsenic content of soils from three regions of Santa Catarina State}

\section{ABSTRACT}

The determination of trace elements is necessary in order to monitor their entry into the soil system and to remediate contaminated areas. The purpose of this study was to determine the natural content of arsenic (As) in soils of three regions of Santa Catarina State (SC): the Southern Plateau, the Metropolitan area and the Southern Coast. Arsenic content was obtained after digestion in a microwave oven, following the USEPA 3051 A protocol and quantification was made by atomic absorption spectrometry with electrothermal atomization. The results were analyzed by the Scott-Knott test at a 5\% significance level. Soil attributes that best correlated with arsenic content were clay, organic carbon, cation exchange capacity and $\mathrm{Al}$ and $\mathrm{Fe}$ oxides. The arsenic levels are related to the source material and the slope of regional soils.

Keywords: contaminated areas, remediation, trace-elements. 


\section{INTRODUÇÃO}

O Arsênio (As) é o vigésimo elemento mais abundante na crosta terrestre e pertence ao grupo V da tabela periódica (Roy et al., 2015). Apresenta alta toxicidade para a saúde humana e animal e está extensamente distribuído na atmosfera, hidrosfera e biosfera, sendo associado tanto a fontes naturais, quanto antropogênicas. São fontes naturais, o intemperismo das rochas, atividades biológicas e emissões vulcânicas (Alonso et al., 2014). As fontes antropogênicas incluem herbicidas, fertilizantes fosfatados, atividades de mineração, resíduos industriais e atividades relacionadas à preservação da madeira (Chirenje et al., 2003; Alonso et al., 2014; Roy et al., 2015).

O As é um elemento calcófilo (Goldschmidt, 1958) e sua mobilidade no solo é regulada pelo seu estado de oxidação (Tarvainen et al., 2013). No solo, em condições óxicas (Eh > $200 \mathrm{mV}$; pH 5-8) o As é encontrado na forma $\mathrm{As}^{5+}$, enquanto que o $\mathrm{As}^{3+}$ é encontrado em condições anóxicas (Mcbride, 1994; Singh et al., 2015). O controle da solubilidade do As na solução do solo em condições aeróbias é feito pelas formas $\mathrm{Ca}_{3}\left(\mathrm{AsO}_{4}\right)_{2}, \mathrm{Mg}_{3}\left(\mathrm{AsO}_{4}\right)_{2}$ e $\mathrm{As}_{2} \mathrm{O}_{5}$ e em condições anaeróbias, $\mathrm{As}, \mathrm{As}_{2} \mathrm{~S}_{3}$ e $\mathrm{As}_{2} \mathrm{O}_{3}$ (Hayes e Traina, 1998).

O comportamento do arsenato no solo é semelhante ao do fosfato e vanadato (Mcbride, 1994; Rosas-Castor et al., 2014). O arsenato sofre adsorção em óxidos de $\mathrm{Fe}$ e $\mathrm{Al}$, aluminossilicatos não cristalinos e, em menor extensão, em argilossilicatos. $\mathrm{O}$ arsenato é o ânion do ácido forte $\mathrm{H}_{3} \mathrm{AsO}_{4}$ (ácido arsênico) que possui valores de pKa 2,24; 6,94 e 11,5; sendo adsorvido efetivamente em $\mathrm{pH}$ baixo (Mcbride, 1994). Os ânions $\mathrm{AsO}_{2}{ }^{-}, \mathrm{AsO}_{4}{ }^{3-}$, $\mathrm{HAsO}_{4}{ }^{2-} \mathrm{e}_{2} \mathrm{AsO}_{3}{ }^{-}$são formas móveis de As, sendo sorvidas em $\mathrm{pH}$ entre 7-9 (Mcbride, 1994). $\mathrm{O} \mathrm{As}^{3+}$ é 10 vezes mais tóxico que o $\mathrm{As}^{5+}$ (Rosas-Castor et al., 2014). O As está classificado pela Agency for Toxic Substances and Disease Registry - ATSDR (ATSDR, 2013) em primeiro lugar como a substância mais perigosa à saúde humana.

A maioria dos humanos está exposta a baixas concentrações de As, principalmente através de ingestão de alimentos e água (Singh et al., 2015). A ingestão de água contaminada com As talvez seja a via mais comum de intoxicação de seres humanos (Fitz e Wenzel, 2002). Entretanto, Zakharova et al. (2002) observaram que a exposição a As via ingestão de produtos agrícolas, contato com a pele e ingestão de solo resultam em alto risco à saúde. A ingestão de solo pode não ser uma via significante de contaminação para os adultos, porém pode ser considerável para crianças, particularmente em locais próximos a indústrias ou a locais que recebem descarte de resíduos de mineração e outros (WHO, 2001).

A importância de se conhecer os teores naturais de elementos-traço no solo, sem interferência humana, é necessária para o monitoramento e a remediação de áreas contaminadas e, além disso, contribui para o entendimento da magnitude dos riscos que a população está exposta diariamente (Tsuji et al., 2007). O acúmulo desses elementos, seja ele causado por atividades agrícolas ou industriais é preocupante, devido a uma possível transferência desses elementos para animais e seres humanos (Su e Yang, 2008).

A litologia e os processos de formação do solo estão interligados com os teores de elementos-traços no solo, sendo comum comparar a composição química do solo com o material de origem na avaliação de um solo contaminado (Parra et al., 2014). Para monitorar a contaminação do solo por um elemento, primeiramente, é necessário determinar a concentração do mesmo no solo em seu estado natural (Tarvainen et al., 2013) e, assim, obter os VRQ (Valor de Referência de Qualidade) dos solos (Biondi et al., 2011; Mendoza-Grimon et al., 2014). Os solos apresentam características específicas determinadas pela geologia, geomorfologia e clima, que determinam os processos pedogenéticos regionais, o que torna inadequado o uso dos valores genéricos para países e áreas diferentes do local de obtenção dos dados (Biondi et al., 2011). 
A determinação dos teores de As no estado de Santa Catarina, que possui uma área territorial de $95.913 \mathrm{~km}^{2}$, representando $16,61 \%$ da Região Sul e 1,11\% da área total do Brasil (Embrapa, 1998) é de suma importância no auxílio da identificação de áreas contaminadas e no gerenciamento das mesmas.

Considerando o exposto, o objetivo desse trabalho foi determinar os teores naturais de As em solos de três regiões do estado de SC sem ação antrópica, que possam ser aplicados em estudos de monitoramento e remediação no Estado.

\section{MATERIAL E MÉTODOS}

A pesquisa foi realizada em três regiões do estado de $\mathrm{SC}$, sendo Planalto Sul, Metropolitana e Litoral Sul (Figura 1).

A classificação das regiões utilizadas nesse estudo é a indicada pela Embrapa (1998) que realizou o levantamento de reconhecimento de solos do estado de SC. Os 14 solos avaliados (Tabela 1) são provenientes de um Banco de Solos da Universidade do Estado de Santa Catarina (UDESC) e foram coletados, descritos e classificados por Paes Sobrinho (2009); Costa (2013); Ferreira (2013); Lunardi Neto e Almeida (2013) e Teske et al. (2013).

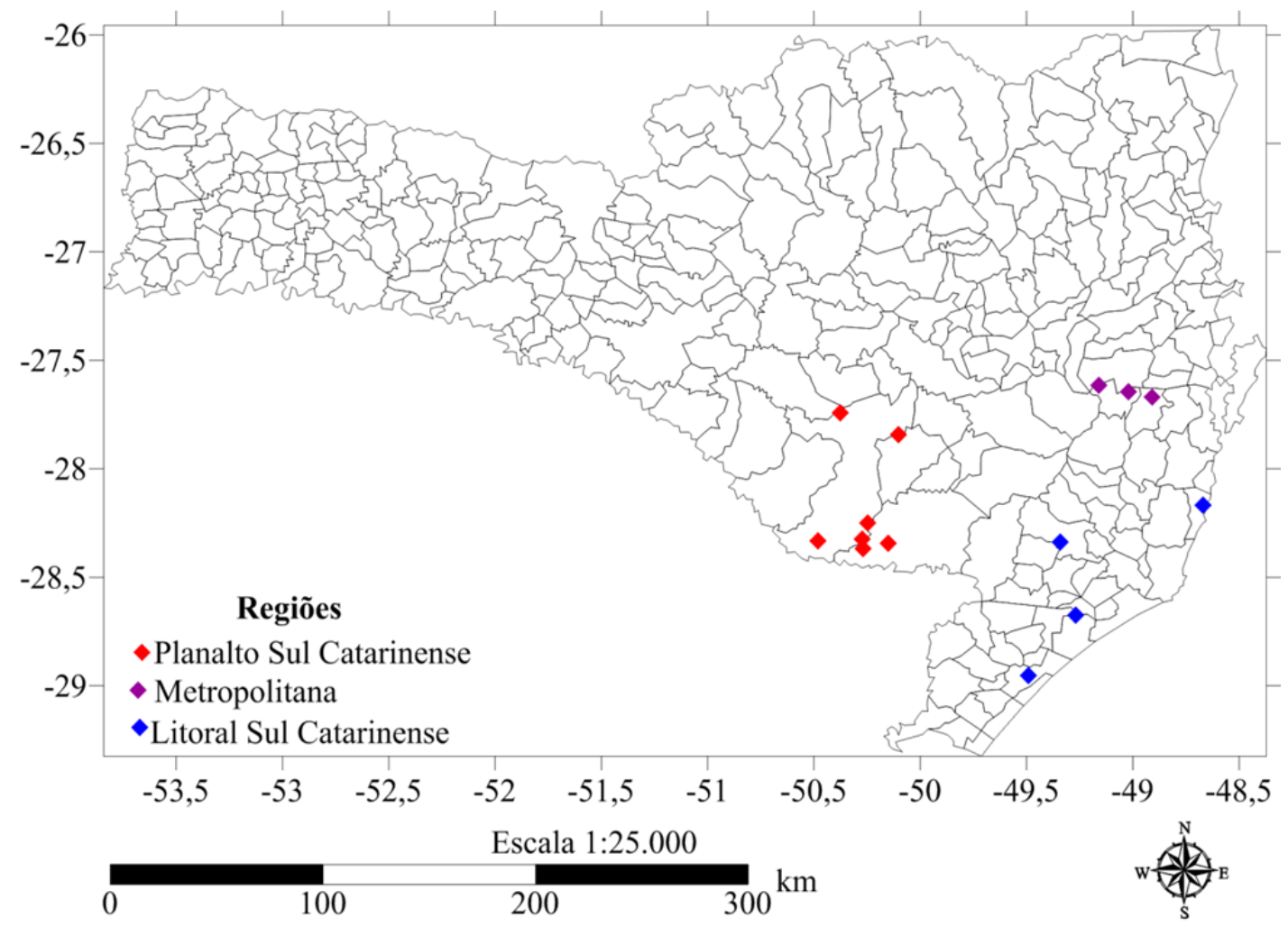

Figura 1. Mapa de Localização das Regiões Planalto Sul, Metropolitana e Litoral Sul no estado de SC.

Os perfis foram descritos e amostrados em corte de barranco de estrada, em locais sob vegetação natural de campo ou de mata, sendo que as amostras utilizadas neste estudo proveram do Horizonte A. Os mesmos autores que realizaram, descreveram e classificaram os solos deste estudo, também determinaram os atributos físicos e químicos dos solos, que se encontram na Tabela 2. 
Tabela 1. Regiões, classes pedológicas, material de origem e localização no Estado.

\begin{tabular}{|c|c|c|c|c|}
\hline Região & Classe & Material de Origem & Localização & $\begin{array}{l}\text { Profundidade } \\
\quad(\mathrm{cm})\end{array}$ \\
\hline \multirow{7}{*}{ Planalto Sul } & Nitossolo Bruno Distrófico típico - $\mathrm{NBd}^{*}$ & Basalto & Painel & $0-17$ \\
\hline & Cambissolo Húmico Distroférrico típico - CHd1 & Basalto & Lages & $0-22$ \\
\hline & Cambissolo Háplico Alumínico úmbrico - CXa & Riodacito & Lages & $0-13$ \\
\hline & Cambissolo Húmico Distrófico típico - CHd2 & Riodacito & Lages & $0-26$ \\
\hline & Nitossolo Vermelho Alítico típico - NVal & Basalto & Lages & $0-12$ \\
\hline & Cambissolo Háplico Alítico típico - Cxal1 & Riodacito & Lages & $0-14$ \\
\hline & Cambissolo Háplico Alítico típico - CXal2 & Riodacito & Lages & $0-12$ \\
\hline \multirow{3}{*}{ Metropolitana } & Argissolo Bruno-Acinzentado Alítico típico - PBACal & Argilitos e Siltitos & $\begin{array}{l}\text { Alfredo } \\
\text { Wagner }\end{array}$ & $0-13$ \\
\hline & Argissolo Amarelo Distrófico típico - PAd & Granito e Granulito & $\begin{array}{l}\text { Rancho } \\
\text { Queimado }\end{array}$ & $0-20$ \\
\hline & $\begin{array}{l}\text { Argissolo Vermelho-Amarelo Distrófico latossólico - } \\
\text { PVAd1 }\end{array}$ & Migmatito & $\begin{array}{l}\text { Águas } \\
\text { Mornas }\end{array}$ & $0-24$ \\
\hline \multirow{4}{*}{ Litoral Sul } & Neossolo Quartzârenico Órtico típico - Rqo1 & Sedimentos Arenosos & Imbituba & $0-28$ \\
\hline & Argissolo Vermelho Distrófico abrúptico - PVd & Siltito e Arenito & Içara & $0-10$ \\
\hline & Neossolo Quartzarênico Órtico típico - Rqo2 & Sedimentos Arenosos & Araranguá & $0-23$ \\
\hline & $\begin{array}{l}\text { Argissolo Vermelho-Amarelo Distrófico latossólico - } \\
\text { PVAd2 }\end{array}$ & Arenito e Siltito & $\begin{array}{l}\text { Lauro } \\
\text { Muller }\end{array}$ & $0-13$ \\
\hline
\end{tabular}

Nota: ${ }^{*}$ Simbologia das Classes de $1^{\circ}, 2^{\circ}$ e $3^{\circ}$ níveis de acordo com a Embrapa (2013).

Tabela 2. Atributos físicos e químicos ${ }^{1}$ e óxidos de $\mathrm{Al}$ e $\mathrm{Fe}^{2}$ dos solos.

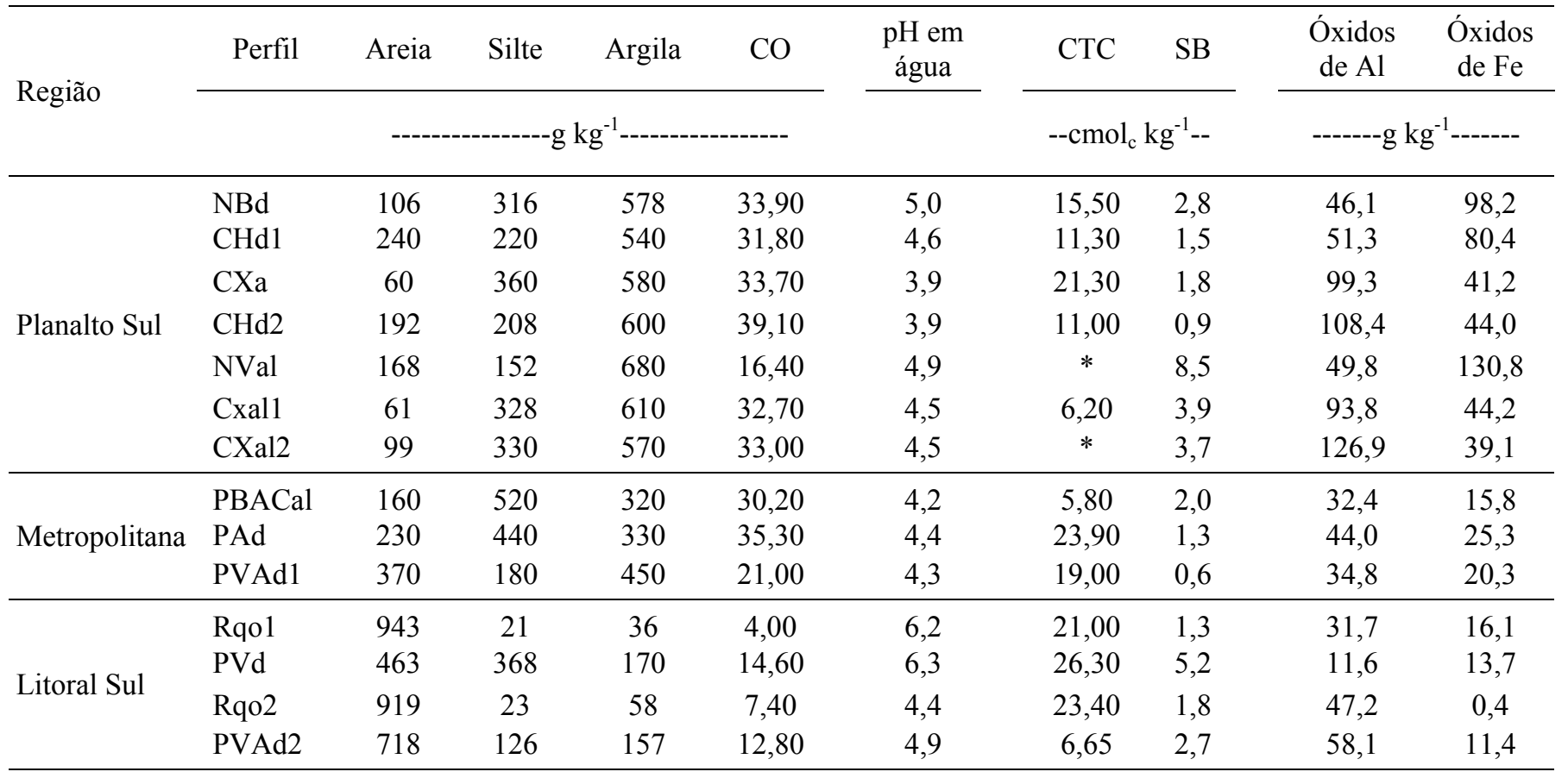

Fontes: ${ }^{1}$ Paes Sobrinho et al. (2009); Costa et al. (2013); Ferreira (2013); Lunardi Neto e Almeida (2013); Teske et al. (2013); conforme metodologia da Embrapa (1999); ${ }^{2}$ próprio autor, conforme Método USEPA 3051 A (USEPA, 1998); *Dados não determinados. CO: Carbono Orgânico; pH: potencial Hidrogeniônico; CTC: Capacidade de Troca de Cátions; SB: Soma de Bases; Al: Alumínio; Fe: Ferro.

As análises foram conduzidas ao Laboratório de Análise Ambiental do Departamento de Solos e Recursos Naturais do Centro de Ciências Agroveterinárias - UDESC/CAV. As amostras, após secas ao ar, foram moídas e passadas em peneira com abertura de $2 \mathrm{~mm}$. Após 
homogeneizadas, as amostras foram maceradas em almofariz de ágata até pó fino e tamisadas em peneira de 0,106 $\mathrm{mm}$ de abertura. Adicionaram-se $0,25 \mathrm{~g}$ de amostra de solo e $6 \mathrm{~mL}$ de solução de $\mathrm{HNO}_{3}$ 14,5 mol L${ }^{-1}$ em tubos de teflon, os quais foram levados para forno de Micro-ondas Multiwave 3000 para a digestão segundo método USEPA 3051 A descrito pela United States Environnmental Protection Agency - USEPA (1998).

Após a digestão e filtragem, o volume final foi completado até $10 \mathrm{~mL}$ com água ultrapura. As análises foram realizadas em duplicata. Em cada bateria foi inserido amostra referência SRM 2709 San Joaquin soil, certificada pelo National Institute of Standards and Technology (NIST) e provas em branco para cálculo do Limite de Detecção Qualitativo do Método Analítico (LDQM) (Tabela 3).

Tabela 3. Teores de As na amostra-controle SRM 2709 determinado e certificado, teores recuperados e LDQM.

\begin{tabular}{|c|c|c|c|}
\hline Teor & \multicolumn{2}{|c|}{ Recuperação } & \\
\hline Determinado Certificado & Determinado & Lixiviado $^{1}$ & LDQM \\
\hline $\mathrm{mg} \mathrm{kg}^{-1}$ & 0 & & \\
\hline $17,7 \pm 0,8$ & 47,7 & ** & 0,48 \\
\hline
\end{tabular}

Notas: ${ }^{1} \mathrm{O}$ certificado NIST SRM 2709 apresenta valores de \% de recuperação para lixiviação ácida para As método USEPA $3050 \mathrm{~B}$ em bloco digestor. **valores não determinados pelo NIST (2002).

O LDQM foi calculado segundo a equação LDQM $=\mathrm{Fd} \times(\mathrm{M} \pm \mathrm{t} \times \mathrm{s})$ (APHA et al., 1998), em que Fd é o fator de diluição das amostras, M é a média das provas em branco, t é o valor t-Student para um intervalo de confiança de $99 \%$, em função do número de graus de liberdade de medições repetidas; e s, o desvio padrão das provas em branco.

Os teores de As foram determinados por espectrofotometria de absorção atômica com atomização eletrotérmica (EAA-FG CONTRAA 700 ANALYTIK JENA). As curvas de calibração foram preparadas a partir de uma solução padrão de $1.000 \mathrm{mg} \mathrm{L}^{-1}$ certificada NIST, marca MERCK, utilizando-se água ultrapura para diluição. A condição de leitura utilizada para As foi a temperatura de pirólise de 700 a $1100^{\circ} \mathrm{C}$ e como modificador químico $\mathrm{Pd}\left(\mathrm{NO}_{3}\right)_{2}$ a $1 \%$ conforme recomendação do fabricante.

Não foi possível comparar a taxa de recuperação porque o protocolo não apresentou a porcentagem recuperada para o método lixiviado para As, método similar ao deste estudo.

As análises estatísticas foram conduzidas utilizando-se o programa Sisvar 5.3 (Ferreira, 2010) e o software $\mathrm{SAS}^{\circledR}$ (SAS Institute, 2003). Os resultados foram submetidos à análise de variância e teste de Scott-Knott. Para todos os testes, foi considerado o nível mínimo de 5\% de significância. Os solos da região Litoral Sul foram excluídos das análises estatísticas, pois todos os resultados analíticos ficaram abaixo do LDQM.

\section{RESULTADOS E DISCUSSÃO}

Os teores de As nesse estudo variaram de $<0,48$ a 9,36 $\mathrm{mg} \mathrm{kg}^{-1}$ (Tabela 4).

Tabela 4. Teores médios de As para as regiões Planalto Sul, Metropolitana e Litoral Sul.

\begin{tabular}{lc}
\hline Região & $\mathrm{As}\left(\mathrm{mg} \mathrm{kg}^{-1}\right)$ \\
\hline Planalto Sul & $6,79 \mathrm{~A}$ \\
Metropolitana & $5,55 \mathrm{~A}$ \\
Litoral Sul & $<0,48$ \\
\hline
\end{tabular}

Nota: As comparações entre as regiões foram testadas pelo teste de Scott-Knott a nível mínimo de 5\% de significância, sendo que médias seguidas da mesma letra não diferem entre si. 
Não houve diferença estatística entre as regiões Planalto Sul e Metropolitana (Tabela 4), enquanto que para a região Litoral Sul os teores de As dos solos ficaram abaixo do LDQM. Isso pode ser explicado pelas diferenças no material de origem e relevo das regiões. Os solos do Litoral Sul são formados por sedimentos recentes de rochas sedimentares, nos quais são encontrados altos teores de areia, que possivelmente não apresentam minerais que contenham As, além da baixíssima capacidade de adsorção química desse material. Outra questão é que os solos do Litoral Sul se encontram em relevo plano e são bem drenados (Costa et al., 2013), o que permite uma maior lixiviação dos elementos presentes nos mesmos.

Quando comparado às classes de solos dentro de cada região, verifica-se que não houve diferença significativa nos teores de As (Tabela 5).

Tabela 5. Teores médios de As dos solos das regiões Planalto Sul e Metropolitana.

\begin{tabular}{|c|c|}
\hline Classe de Solo & As $\left(\mathrm{mg} \mathrm{kg}^{-1}\right)$ \\
\hline \multicolumn{2}{|c|}{ Planalto Sul } \\
\hline NBd & $5,65 \mathrm{~A}$ \\
\hline CHd1 & $8,60 \mathrm{~A}$ \\
\hline $\mathrm{CXa}$ & $6,37 \mathrm{~A}$ \\
\hline $\mathrm{CHd} 2$ & $5,52 \mathrm{~A}$ \\
\hline NVal & $9,36 \mathrm{~A}$ \\
\hline CXall & $8,38 \mathrm{~A}$ \\
\hline CXal2 & $6,62 \mathrm{~A}$ \\
\hline \multicolumn{2}{|c|}{ Metropolitana } \\
\hline PBACal & $4,60 \mathrm{~A}$ \\
\hline PVA & $7,27 \mathrm{~A}$ \\
\hline PVAd1 & $4,78 \mathrm{~A}$ \\
\hline
\end{tabular}

Nota: As comparações entre classes de solos dentro de cada região foram testadas pelo teste de Scott-Knott a nível mínimo de 5\% de significância, sendo que médias seguidas da mesma letra não diferem entre si.

$\mathrm{Na}$ análise de correlação (Tabela 6) os teores de As tiveram correlação significativa e positiva com a argila $(0,89), \mathrm{CO}(0,72)$ e os óxidos de ferro $(0,74)$. Segundo Mcbride $(1994)$ e Kabata-Pendias (2011) a mobilidade desse elemento é reduzida devido à forte sorção por argilas, hidróxidos e matéria orgânica.

Tabela 6. Correlação entre os atributos físicos e químicos e os teores de As.

\begin{tabular}{cccccccccc}
\hline & Areia & Silte & Argila & $\mathrm{CO}$ & $\begin{array}{c}\mathrm{pH} \\
\text { em água }\end{array}$ & CTC & SB & $\begin{array}{c}\text { Óxidos } \\
\text { de Al }\end{array}$ & $\begin{array}{c}\text { Oxidos } \\
\text { de Fe }\end{array}$ \\
\hline As & $-0,86^{* *}$ & $\mathrm{~ns}$ & $0,89^{* *}$ & $0,72^{* *}$ & $\mathrm{~ns}$ & $0,59^{*}$ & $\mathrm{~ns}$ & $0,67^{* *}$ & $0,74^{* *}$ \\
\hline
\end{tabular}

Notas: **significativo ao nível de $1 \%$ de probabilidade $(\mathrm{p}<0,01)$; *significativo ao nível de $5 \%$ de probabilidade $(0,01 \leq \mathrm{p} \leq 0,05)$ e ns não significativo $(\mathrm{p} \geq 0,05)$; $\mathrm{CO}$ : Carbono Orgânico; $\mathrm{pH}$ : potencial Hidrogeniônico; CTC: Capacidade de Troca de Cátions; SB: Soma de Bases; Al: Alumínio; Fe: Ferro.

Segundo Wang e Mulligan (2006), a matéria orgânica pode aumentar a mobilidade de As, pois eles competem pelos sítios de adsorção disponíveis, no entanto, ácidos orgânicos podem reduzir a mobilidade, sendo agentes de ligação ou formando complexos insolúveis, especialmente quando saturado com cátions. $\mathrm{O}$ As tem alta afinidade por óxidos, motivo pelo qual houve significativa correlação entre As e óxidos de ferro. Segundo Chirenje et al. (2003), que obtiveram o mesmo comportamento para solos dos Estados Unidos, os óxidos geram superfícies reativas, em que o As é adsorvido, formando compostos estáveis e imóveis. Em 
geral, solos derivados de rochas ricas em óxidos de ferro, como o basalto, possuem maiores teores de As, devido à forte adsorção do elemento aos óxidos de Fe. Correlação significativa e positiva $(0,59)$ foi encontrada com a CTC. Isso ocorre devido à afinidade do oxiânion As de se ligar nos sítios disponíveis na CTC. Dudka e Market (1992) também encontraram correlação de $0,75^{* *}$ com a CTC.

Na região Planalto Sul ocorrem solos derivados de rochas ígneas, que originam solos mais argilosos, com maior capacidade de adsorver o As. São solos menos lixiviados, pois o relevo é suave ondulado a forte ondulado e moderadamente drenado (Costa et al., 2013; Ferreira, 2013; Teske et al., 2013).

Campos et al (2013) encontraram teores de 2,03 $\mathrm{mg} \mathrm{kg}^{-1}$ para solos do Bioma Cerrado. Biondi (2010) encontrou teor médio de As de $0,44 \mathrm{mg} \mathrm{kg}^{-1}$ para solos do Estado de Pernambuco. E para solos do Espírito Santo o teor médio determinado foi de $6,80 \mathrm{mg} \mathrm{kg}^{-1}$ (Paye et al., 2010). Para solos da Cuba, Alfaro et al. (2015) obtiveram 10,8 mg kg-1 de As. Dantu (2010) encontrou 10,65 $\mathrm{mg} \mathrm{kg}^{-1}$ de As para solos na Índia.

Essas diferenças dos teores de As encontrados para os solos de SC em relação a outros Estados brasileiros e a outros estudos encontrados na literatura internacional se deve às peculiaridades locais, como a composição do material de origem, a influência dos processos pedogenéticos e aos atributos físicos e químicos dos solos, que são intrínsecos para cada ambiente. Por isso, é essencial que os Estados brasileiros e os países estabeleçam seus VRQ.

\section{CONCLUSÃO}

Não houve diferença entre as classes de solos para as regiões Planalto Sul e Metropolitana, sendo que na Região Litoral Sul os teores ficaram abaixo do LDQM.

Os teores de As correlacionaram-se positivamente com os teores de argila, CO, CTC e óxidos de $\mathrm{Al}$ e de Fe.

\section{REFERÊNCIAS}

ALFARO, M. R.; MONTERO, A.; UGARTE, O. M.; NASCIMENTO, C.W. A. do; ACCIOLY, A. M. de A.; BIONDI, C. M. et al. Background concentrations and reference values for heavy metals in soils of Cuba. Environmental Monitoring Assessment,. v. 187, p. 1-10, 2015. http://dx.doi.org /10.1007/s10661-014-4198-3

ALONSO, D. L.; LATORRE, S.; CASTILlO, E.; BRANDÃO, P. F. B. Environmental occurrence of arsenic in Colombia: A review. Environmental Pollution, v. 186, p. $272-$ 281, 2014. http://dx.doi.org/10.1016/j.envpol.2013.12.009

AMERICAN PUBLIC HEALTH ASSOCIATION - APHA; AMERICAN WATER WORKS ASSOCIATION - AWWA; WATER ENVIRONMENT FEDERATON - WEF. Standard Methods for the examination of water and wastewater. $20^{\text {th }} \mathrm{ed}$. Washington, DC, 1998.

AGENCY FOR TOXIC SUBSTANCES AND DISEASE CONTROL - ATSDR. Priority list of hazardous substances. 2013. Disponível em: http://www.atsdr.cdc.gov/spl/. Acesso em: 13 jan. 2015.

BIONDI, C. M. Teores naturais de metais pesados nos solos de referência do Estado de Pernambuco. 2010. 70f. Tese (Doutorado em Ciência do Solo) - Universidade Federal Rural de Pernambuco, Recife, 2010. 
BIONDI, C. M.; NASCIMENTO, C.W.A.; NETA, A. B. F.; RIBEIRO, M. R. Teores de Fe, $\mathrm{Mn}, \mathrm{Zn}, \mathrm{Cu}$, Ni e $\mathrm{Co}$ em solos de referência de Pernambuco. Revista Brasileira de Ciência do Solo, v. 35, p. 1057-1066, 2011. http://dx.doi.org/10.1590/s010006832011000300039

CAMPOS, M. L.; GUILHERME, L. R. G.; MARQUES, J. J. G. de S. e M.; CURI, N.; ARAÚJO, A. S. A. A.; MIQUELLUTI, D. J. et al. Teores de arsênio e cádmio em solos do bioma cerrado. Revista Brasileira de Ciência do Solo, v. 37, n. 1, p. 281-286, 2013. http://dx.doi.org/10.1590/s0100-06832013000100029

CHIRENJE, T.; MA, L. Q.; CHEN, M.; ZILLIOUX, E. J. Comparison between background concentrations of arsenic In urban and non-urban areas of Florida. Advances in Environmental Research, v. 8, p. 137-146, 2003. http://dx.doi.org/10.1016/s1093$0191(02) 00138-7$

COSTA, A. da.; ALBUQUERQUE, J. A.; ALMEIDA, J. A.; COSTA, A. da.; LUCIANO, R. V. Pedotransfer functions to estimate retention and availability of water in soils of the state of Santa Catarina, Brazil. Revista Brasileira de Ciência do Solo, v. 37, p. 889910, 2013. http://dx.doi.org/10.1590/s0100-06832013000400007

DANTU, S. Geochemical patterns in soils in and around Siddipet, Medak District, Andhra Pradesh, India. Environmental Monitoring Assessment, v. 170, p. 681-701, 2010. http://dx.doi.org/10.1007/s10661-009-1267-0

DUDKA, S.; MARKERT, B. Baseline Concentrations of As, Ba, Be, $\mathrm{Li}, \mathrm{Nb}, \mathrm{Sr}$, and $\mathrm{V}$ in surface soils of Poland. The Science of the total Environment, v. 22, p. 279-290, 1992. http://dx.doi.org/10.1016/0048-9697(92)90046-u

EMPRESA BRASILEIRA DE PESQUISA AGROPECUÁRIA - EMBRAPA. Levantamento de Reconhecimento dos Solos do Estado de Santa Catarina. Rio de Janeiro, 1998. 735p (Boletim de Pesquisa, 6).

EMPRESA BRASILEIRA DE PESQUISA AGROPECUÁRIA - EMBRAPA. Manual de métodos de análise de solo. 2. ed. Rio de Janeiro, 1999. 212 p.

EMPRESA BRASILEIRA DE PESQUISA AGROPECUÁRIA - EMBRAPA. Sistema Brasileiro de Classificação de Solos. 3. ed. Brasília, 2013. 353p.

FERREIRA, D. F. SISVAR 5.3. Sistema de Análises Estatísticas. 2010. Disponível em: http://www.dex.ufla.br/. Acesso em: 10 jan. 2015.

FERREIRA, E. R. N. C. Química e Mineralogia de solos desenvolvidos de rochas alcalinas e ultrabásicas do Domo de Lages. 2013. 159f. Tese (Doutorado em Manejo do Solo) - Universidade do Estado de Santa Catarina, Lages, 2013.

FITZ, W. J.; WENZEL, W. W. Arsenic transformations in soil-rhizosphere-plant system: fundamentals and potential application to phytoremediation. Journal of Biotechnology, v. 99, p. 259-278, 2002. http://dx.doi.org/10.1016/s0168-1656(02)00218-3

GOLDSCHMIDT, V. M. Geochemistry. Londres: Oxford University Press, 1958. 425 p.

HAYES, K. F.; TRAINA, S. J. Metal speciation and its significance in ecosystem health. In: HUANG, P. M. Soil chemistry and ecosystem health. Madison: Soil Science Society of America Journal, 1998. p. 45-84. 
KABATA-PENDIAS, A. Trace elements in soils and plants. Boca Raton: CRC Press, 2011. $315 \mathrm{p}$.

LUNARDI NETO, A.; ALMEIDA, J. A. Mineralogia das frações silte e argila em Argissolos com horizontes subsuperficiais escurecidos em Santa Catarina. Revista de Ciências Agroveterinárias, v. 12, p. 282-293, 2013. http://dx.doi.org/10.1590/s010006832012000400001

MCBRIDE, M. B. Environmental chemistry of soils. New York: Oxford University Press, 1994. $406 \mathrm{p}$.

MENDOZA-GRIMÓN, V.; HERNÁNDEZ-MORENO, J. M.; MARTÍN, J. A. R.; FERNÁNDEZ-VERA, J. R.; PALACIOS-DÍAZ, M. P. Trace and major element associations in basaltic ash soils of El Hierro Island, Journal of Geochemical Exploration, v. 147, p. 277-282, 2014. http://dx.doi.org/10.1016/j.gexplo.2014.06.010

NATIONAL INSTITUTE OF STANDARDS AND TECHNOLOGY STANDARD REFERENCE MATERIALS - NIST. SRM 2709, 2710 and 2711. 2002. Disponível em: https://www-s.nist.gov/srmors/certificates/archive/2709.pdf. Acesso em: 10 jan. 2012.

PAYE, H. S.; MELLO, J. W. V.; ABRAHÃO, W. A. P.; FERNANDES FILHO, E. I.; DIAS, L. C. P.; CASTRO, M. L. O. et al. Valores de referência de qualidade para metais pesados em solos no Estado do Espírito Santo. Revista Brasileira de Ciência do Solo, v. 34, p. 2041-2051, 2010. http://dx.doi.org/10.1590/s0100-06832010000600028

PAES SOBRINHO, J. B.; ALMEIDA, J. A.; ERHART, J. Mineralogia da fração argila de Argissolos das Serras do Leste de Santa Catarina. Revista de Ciências Agroveterinárias, v. 8, p. 09-24, 2009.

PARRA, S.; BRAVO, M. A.; QUIROZ, W.; MORENO, T.; KARANASIOU, A.; FONT, O. et al. Distribution of trace elements in particle size fractions for contaminated soils by a copper smelting from different zones of the Puchuncaví Valley (Chile). Chemosphere, v. 111, p. 513-521, 2014. http://dx.doi.org/10.1016/j.chemosphere.2014.03.127

ROSAS-CASTOR, J. M.; GUZMÁN-MAR, J. L.; HERNÁNDEZ-RAMÍREZ, A.; GARZAGONZÁLEZ, M. T.; HINOJOSA-REYES, L. Arsenic accumulation in maize crop (Zea mays): A review. Science of the Total Environment, v. 488-489, p. 176-187, 2014. http://dx.doi.org/10.1016/j.scitotenv.2014.04.075

ROY, M.; GIRI, A. K.; DUTTA, S.; MUKHERJEE, P. Integrated phytobial remediation for sustainable management of arsenic in soil and water. Environment International, v. 75, p. 180-198, 2015. http://dx.doi.org/10.1016/j.envint.2014.11.010

SAS INSTITUTE INC ${ }^{\circledR}$ Statistical Analysis System. Versão 9.1.3. Cary, 2003. 1 CD ROM.

SINGH, R.; SINGH, S.; PARIHAR, P.; SINGH, V. P.; PRASAD, S. M. Arsenic contamination, consequences and remediation techniques: a review. Ecotoxicology and Environmental Safety, v. 112, p. 247-270, 2015.

http://dx.doi.org/10.1016/j.ecoenv.2014.10.009

SU, Y.; YANG, R. Background concentrations of elements in surface soils and their changes as affected by agriculture use in the desert-oasis ecotone in the middle of Heihe River Basin, North-west China. Journal of Geochemical Exploration, v. 98, p. 57-64, 2008. http://dx.doi.org/10.1016/j.gexplo.2007.12.001 
TARVAINEN, T.; ALBANESE, S.; BIRKE, M.; PONAVIC, M.; REIMANN, C. Arsenic in agricultural and grazing land soils of Europe. Applied Geochemistry, v. 28, p. 2-10, 2013. http://dx.doi.org/10.1016/j.apgeochem.2012.10.005

TESKE, R.; LUNARDI NETO, A.; HOFER, A.; ALMEIDA, J. A. Caracterização química, física e morfológica de solos derivados de rochas efusivas no planalto sul de Santa Catarina, Brasil. Revista de Ciências Agroveterinárias, v. 12, p. 175-186, 2013.

TSUJI, J. S.; YOST, L. J.; BARRAJ, L. M, SCRAFFORD, C. G.; MINK, P. J. Use of background inorganic arsenic exposures to provide perspective on risk assessment results. Regulatory Toxicology and Pharmacology, v. 48, p. 59-68, 2007. http://dx.doi.org/10.1016/j.yrtph.2007.01.004

UNITED STATES. Environmental Protection Agency - USEPA. Test Methods for Evaluation Solid Waste Physical and Chemical Methods: microwave assisted acid digest of sediments, sludges, soils and oils, SW 846; U. S. Washington: Government Printing Office, 1998.

WANG, S.; MULLIGAN, C. N. Effect of natural organic matter on arsenic release from soils and sediments into groundwater. Environmental and Health, v. 28, p. 197-214, 2006. http://dx.doi.org/10.1007/s10653-005-9032-y

WORLD HEALTH ORGANIZATION - WHO. Arsenic and arsenic compounds. 2001. Disponível em: http://www.who.int/ipcs/publications/ehc/ehc_224/en/ Acesso em: 12 jan. 2015.

ZAKHAROVA, T.; TATANO, F.; MENSHIKOV, V. Health cancer risk assessment for arsenic exposure in potentially contaminated areas by plants fertilizer: A possible regulatory approach applied to a case study in Moscow Region-Russia. Regulatory $\begin{array}{llllll}\text { Toxicology and Pharmacology, v. 36, p. 22-23, } 2002 . & \end{array}$ http://dx.doi.org/10.1006/rtph.2002.1561 